\title{
Improved Prognostic Stratification of Patients With pN3b Oral Cavity Cancer Based on Maximum Standardized Uptake Value of Metastatic Nodes, Lymph Node Ratio, and Level of Cervical Nodal Metastases
}

\author{
Nai-Ming Cheng \\ Chang Gung Memorial Hospital Linkou Main Branch: Chang Gung Memorial Hospital \\ Chung-Jan Kang \\ Chang Gung Memorial Hospital Linkou Main Branch: Chang Gung Memorial Hospital \\ Chi-Ying Tsai \\ Chang Gung Memorial Hospital Linkou Main Branch: Chang Gung Memorial Hospital \\ Lan-Yan Yang \\ Chang Gung Memorial Hospital Linkou Main Branch: Chang Gung Memorial Hospital \\ Li-Yu Lee \\ Chang Gung Memorial Hospital Linkou Main Branch: Chang Gung Memorial Hospital \\ Chien-Yu Lin \\ Chang Gung Memorial Hospital Linkou Main Branch: Chang Gung Memorial Hospital \\ Chuen Hsueh \\ Chang Gung Memorial Hospital Linkou Main Branch: Chang Gung Memorial Hospital \\ Kang-Hsing Fan \\ Chang Gung Memorial Hospital Linkou Main Branch: Chang Gung Memorial Hospital \\ Hung-Ming Wang \\ Chang Gung Memorial Hospital Linkou Main Branch: Chang Gung Memorial Hospital \\ Chia-Hsun Hsieh \\ Chang Gung Memorial Hospital Linkou Main Branch: Chang Gung Memorial Hospital \\ Shu-Hang Ng \\ Chang Gung Memorial Hospital Linkou Main Branch: Chang Gung Memorial Hospital \\ Chih-Hua Yeh \\ Chang Gung Memorial Hospital Linkou Main Branch: Chang Gung Memorial Hospital \\ Chih-Hung Lin \\ Chang Gung Memorial Hospital Linkou Main Branch: Chang Gung Memorial Hospital \\ Chung-Kan Tsao \\ Chang Gung Memorial Hospital Linkou Main Branch: Chang Gung Memorial Hospital \\ Tuan-Jen Fang \\ Chang Gung Memorial Hospital Linkou Main Branch: Chang Gung Memorial Hospital \\ Shiang-Fu Huang \\ Chang Gung Memorial Hospital Linkou Main Branch: Chang Gung Memorial Hospital \\ Li-Ang Lee \\ Chang Gung Memorial Hospital Linkou Main Branch: Chang Gung Memorial Hospital \\ Ku-Hao Fang \\ Chang Gung Memorial Hospital Linkou Main Branch: Chang Gung Memorial Hospital \\ Yu-Chien Wang \\ Chang Gung Memorial Hospital Linkou Main Branch: Chang Gung Memorial Hospital \\ Wan-Ni Lin \\ Chang Gung Memorial Hospital Linkou Main Branch: Chang Gung Memorial Hospital \\ Li-Jen Hsin
}

Page $1 / 15$ 
Chang Gung Memorial Hospital Linkou Main Branch: Chang Gung Memorial Hospital

Tzu-Chen Yen

Chang Gung Memorial Hospital Linkou Main Branch: Chang Gung Memorial Hospital

Chun-Ta Liao ( $\square$ liaoct@cgmh.org.tw)

Chang Gung Memorial Hospital Linkou Main Branch: Chang Gung Memorial Hospital

\section{Research Article}

Keywords: Oral cavity squamous cell carcinoma, FDG-PET, lymph node ratio, level IV/V metastases, pN3 disease

Posted Date: April 21st, 2021

DOI: https://doi.org/10.21203/rs.3.rs-416826/v1

License: @ (i) This work is licensed under a Creative Commons Attribution 4.0 International License. Read Full License

Version of Record: A version of this preprint was published at Oral Oncology on December 1st, 2021. See the published version at https://doi.org/10.1016/j.oraloncology.2021.105593. 


\section{Abstract \\ Objective}

We sought to improve the prognostic stratification of patients with pN3b oral cavity squamous cell carcinoma (OCSCC) through a combined analysis of FDG-PET parameters and clinicopathological risk factors (RFs).

\section{Methods}

Complete data on maximum standardized uptake values derived from FDG-PET of neck metastatic nodes (SUV-nodal-max) and clinicopathological RFs were available for 257 patients with pN3b disease. Participants with pN0/pN1/pN2 ( $n=1318 / 199 / 213)$ disease were included for comparison purposes.

\section{Results}

Using the 5-year disease-free survival (DFS) rate as the outcome of interest, the optimal cutoff points for SUV-nodal-max and lymph node ratio (LNR) were 15.9 and 0.17, respectively. After stratification with SUV-nodal-max and clinicopathological RFs, the 5-year DFS rates for patients with pN3 disease were as follows: SUV-nodal-max $<15.9(\mathrm{n}=226)$ versus SUV-nodal-max $\geq 15.9(\mathrm{n}=31), 49 \% / 21 \%, p=0.000003$; LNR $<0.17(n=230)$ versus $L N R \geq 0.17(n=27), 49 \% / 17 \%, p=0.000117$; absence $(n=230)$ versus presence $(n=27)$ of neck level IV/V metastases, $49 \% / 15 \%, p=0.000004$. Multivariable analyses revealed that SUV-nodal-max $\geq 15.9, \mathrm{LNR} \geq 0.17$, and the presence of level IV/V metastases were independent prognosticators for 5-year distant metastases (DM), DFS, disease-specific survival (DSS) and overall survival (OS) rates. Based on these variables, we devised a scoring system that identified three distinct prognostic subgroups of low (score $0, n=190$ ), intermediate (score $1, n=51$ ), and high (scores $2-3, n=16$ ) risk. The 5 -year rates of $p N 0 / p N 1 / p N 2 / p N 3 b$ of our cohort and the low/intermediate/high risk subgroups of pN3b were as follows: DM, $3 \% / 12 \% / 20 \% / 38 \%$ and $31 \% / 52 \% / 89 \%$; DFS, $83 \% / 72 \% / 65 \% / 46 \%$ and $54 \% / 26 \% / 0 \%$; DSS, $92 \% / 79 \% / 71 \% / 52 \%$ and $59 \% / 36 \% / 8 \%$; OS, $81 \% / 62 \% / 54 \% / 38 \%$ and $42 \% / 31 \% / 6 \%$, respectively; all $p<0.001$.

\section{Conclusion}

A scoring system based on maximum SUV-nodal, LNR, and level IV/V metastases helps improve the prognostic stratification of OCSCC patients with pN3 disease.

\section{Introduction}

The traditional therapeutic approach for oral cavity squamous cell carcinoma (OCSCC) relies on radical surgery either with or without adjuvant therapy. The main determinants of clinical outcomes in patients with OCSCC include loco-regional and distant control. The presence and extent of cervical lymph node (LNs) metastases play a crucial role in prognostic stratification and subsequent treatment decisions. However, the clinical course of patients with neck LNs metastases remains heterogeneous - ultimately suggesting that additional prognostic refinements are necessary [1]. Numerous risk factors (RFs) have been identified as independent prognostic variables in patients with OCSCC and neck LNs metastases. They include the number of pathologically positive LNs (pN+) $[2,3]$, the LN ratio (LNR, defined as the number of pathologically positive nodes divided by the number of dissected nodes) [3-10], the log odds of positive LNs (LODDS) [10], and the level of cervical LNs metastases (with level IV/V metastases being generally associated with an unfavorable prognosis) $[1,11,12]$. The presence of distant failure is generally associated with dismal outcomes (mean survival in Taiwanese patients: 2.4 months) [13]. While the distant metastasis (DM) rate of patients with OCSCC and extracapsular extension (ENE) may be as high as $30 \%$, only $1 \%$ of all cases with distant spread are correctly identified during the preoperative workup [14].

Compared with the previous 2010 edition [15], the 2018 AJCC staging manual introduced a novel nodal classification for OCSCC termed pN3b disease - which comprises pN2 disease (defined according to the 2010 edition) and ENE [16].

While this novel classification was aimed to improve prognostic stratification, our pilot clinical experience revealed that the clinical outcomes of patients with pN3b disease are characterized by significant interindividual differences. Starting from these premises, this study was undertaken to further refine the prognostic stratification of patients with pN3b OCSCC through a combined analysis of FDG-PET parameters (collected as of 2001) and clinicopathological RFs (collected as of 1996). 


\section{Patients And Methods \\ Study setting}

We retrospectively reviewed the clinical records of all patients diagnosed with first primary OCSCC $(n=2256)$ who were consecutively referred to the Chang Gung Memorial Hospital (CGMH) from January 1996 to February 2019. Between August 2001 and February 2019 , a total of 1310 patients underwent preoperative FDG-PET imaging. Owing to the prospective collection of data on tumor depth of invasion (DOI) and ENE, patients were staged according to the AJCC staging manual, eighth edition [16]. Patients who did not undergo neck dissection (ND, $n=133$ ), with unavailable data on ENE $(n=3)$, and who had pN3b disease but did not undergo FDG-PET imaging ( $n=133$ ) were excluded - resulting in a final cohort comprising 1987 cases. While the focus of this study was on the prognostic stratification of pN3 disease, patients with pNO - 2 disease were included for comparison purposes. The distribution of pathological nodal stage was as follows: $p N 0, n=1318 ; p N 1, n=199 ; p N 2, n=213$; and pN3b, $n=257$, respectively. We were unable to identify any case with pN3a disease. Figure 1 depicts the flow of participants through the study. The presurgical evaluation and staging workup have been previously described in detail [1]. Clinicopathological RFs were collected in a prospective fashion by investigators blinded to clinical outcomes. In accordance with the recommendations set forth by the CAP Cancer Reporting Protocol [17], a dedicated checklist was used by two experienced headand-neck pathologists to independently review histopathological data. The study protocol was granted ethical approval by the local Institutional Review Board (references: CGMH 101-4457B and 202100048B0). The requirement for written consent was waived due to the study design.

\section{FDG-PET and PET/CT imaging protocols}

A minimum 6-h fast was recommended for all patients before intravenous injection of 370-444 MBq (10-12 mCi) ${ }^{18} \mathrm{~F}-\mathrm{FDG}$. Before April 2006, all participants underwent PET imaging (ECAT EXACT HR_PET camera; CTI, Knoxville, TN, USA). As of May 2006, we used a combined PET/CT scanner (Discovery ST; GE Healthcare, Waukesha, WI, USA). Oral contrast administration was performed during the uptake time of PET/CT, whereas we used no intravenous contrast agent for CT imaging. All patients underwent head to mid-thigh scans. PET and CT data were consecutively acquired 50 min following ${ }^{18} \mathrm{~F}$-FDG injection. PET image reconstruction was carried out using an ordered-subset expectation maximization algorithm. A nuclear medicine team not blinded to patient clinical data reviewed all images in a prospective fashion. ${ }^{18}$ F-FDG-avid foci were thoroughly identified and recorded. All images showing at least one area of increased ${ }^{18} \mathrm{~F}-\mathrm{FDG}$ uptake (i.e., with an intensity higher than that of the surrounding tissue) were considered as positive - provided that the foci did not correspond to areas of physiological tracer distribution on hybrid images. Regions of interest (ROIs) were positioned over visible lesions, as well as on simultaneously displayed axial, coronal, and sagittal images. Maximum standardized uptake value (SUV-max) was defined as the highest activity concentration per injected dose per body weight $(\mathrm{kg})$ after correction for radioactive decay. SUV-max values of the primary tumor and neck lymph nodes (SUV-nodal-max) were calculated in accordance with a previously described methodology [18].

\section{Surgery and adjuvant therapy}

Primary tumors were removed with $\geq 1 \mathrm{~cm}$ margins (both peripheral and deep margins). Patients with $\mathrm{cN}+$ disease underwent level I-IV or I-V NDs, whereas cN- patients received level I-III NDs. Patients who harbored pathological RFs were generally treated with post-operative radiotherapy (RT, $60 \mathrm{~Gy}$ ) or concurrent chemoradiotherapy (CCRT, $66 \mathrm{~Gy}$ ) [19-21]. RFs were assessed using the National Comprehensive Cancer Network (NCCN) guidelines until 2008 [22]; thereafter, the CGMH guidelines were adopted [23]. The radiation field consisted of the entire tumor bed area (with 1- to 2- cm margins) and regional lymphatics. We used the following chemotherapy regimens: intravenous cisplatin $50 \mathrm{mg} / \mathrm{m}^{2}$ biweekly plus daily oral tegafur $800 \mathrm{mg}$ and leucovorin $60 \mathrm{mg}$, cisplatin $40 \mathrm{mg} / \mathrm{m}^{2}$ weekly, or cisplatin $100 \mathrm{mg} / \mathrm{m}^{2}$ every three weeks [21). Patients who refused the proposed approaches or had unexpected evidence of disease stage modifications in the postoperative period were treated with surgery alone.

\section{Follow-up schedule and data collection}

Postoperative follow-up visits were performed at predefined time intervals according to the date of surgery, according to the following scheme: every 1-3 months throughout the first postoperative year; every 2-4 months throughout the second year; and every 4-6 months between the third and the fifth years. Patients who survived for more than 5 years were followed every 6-12 months. At each visit, data on the following clinical events were collected: local control (LC), neck control (NC), DM, disease-free survival (DFS), disease-specific survival (DSS), and overall survival (OS).

\section{Statistical analysis}

The study variables are summarized using descriptive statistics - including frequencies, percentages, means, medians, ranges, and standard deviations (SD). All participants were followed up until February 2021 (minimum follow-up duration after surgery: 24 months). 
The study endpoints included the 5-year rates of LC, NC, DM, DFS, DSS, and OS. All outcomes were calculated as the time from the date of surgery to the date of the event of interest. We calculated Kaplan-Meier probabilities to estimate time to each event, and a log-rank test was performed to assess differences between the groups. To evaluate the association between RFs and clinical outcomes, we performed univariable and multivariable Cox proportional hazard regression analyses. All variables examined at the univariable level were entered as covariates into the multivariable model. We censored for patient death and study completion in outcome analyses. Results are presented as hazard ratios (HRs) with their 95\% confidence intervals (Cls). For all analyses, statistical significance was defined as a two-tailed $p$ value $<0.05$

\section{Results}

\section{Optimal cutoff values for SUV-nodal-max and lymph node ratio in patients with pN3b disease}

A total of 16 variables - including SUV-nodal-max, LNR, and level IV/V metastases - were analyzed in relation to clinical outcomes of patients with pN3b disease. Using the 5-year DFS rate as the endpoint of interest, the optimal cutoff values for the SUV-nodal-max and LNR were $15.9(<15.9$ [ $\mathrm{n}=226$ ] versus $\geq 15.9$ [ $\mathrm{n}=31$ ], 49\%/21\%, $p=0.000003)$ and $0.17(<0.17$ [ $\mathrm{n}=230]$ versus $\geq 0.17$ [ $\mathrm{n}=27$ ], $49 \% / 17 \%$, $p=0.000117$, (Fig. 2A-B), respectively.

\section{General characteristics of patients with OCSCC and pN3 disease}

Table 1 depicts the general characteristics of patients with pN3 disease. 
Table 1

General characteristics of patients with OCSCC and pN3b disease stratified according to SUV-nodalmax $<15.9$ versus $\geq 15.9$ and lymph node ratio $<0.17$ versus $\geq 0.17$

\begin{tabular}{|c|c|c|c|c|c|c|}
\hline \multirow[b]{2}{*}{ Characteristic } & \multicolumn{3}{|c|}{ SUV-nodal-max } & \multicolumn{3}{|c|}{ Lymph node ratio } \\
\hline & $<15.9$ & $\geq 15.9$ & $p$ & $<0.17$ & $\geq 0.17$ & $p$ \\
\hline & $(n=226)$ & $(n=31)$ & & $(n=230)$ & $(n=27)$ & \\
\hline & n (\%) & n (\%) & & $\mathrm{n}(\%)$ & $\mathrm{n}(\%)$ & \\
\hline Lymph node ratio & & & 0.087 & & & \\
\hline$<0.17(230,89.5)$ & $206(90.7)$ & $24(80.0)$ & & & & \\
\hline$\geq 0.17(27,10.5)$ & $21(9.3)$ & $6(20.0)$ & & & & \\
\hline Level IV/V metastases & & & 0.087 & & & 0.001 \\
\hline Absence $(230,89.5)$ & $206(90.7)$ & $24(80.0)$ & & $211(91.7)$ & $19(70.4)$ & \\
\hline Presence $(27,10.5)$ & $21(9.3)$ & $6(20.0)$ & & $19(8.3)$ & $8(29.6)$ & \\
\hline Sex & & & 0.139 & & & 0.713 \\
\hline Male $(242,94.2)$ & $212(93.4)$ & $30(100.0)$ & & $217(94.3)$ & $25(92.6)$ & \\
\hline Female $(15,5.8)$ & $15(6.6)$ & 0 & & $13(5.7)$ & $2(7.4)$ & \\
\hline Age (years) & & & 0.935 & & & 0.004 \\
\hline$<65(225,87.5)$ & $199(87.7)$ & $26(86.7)$ & & $206(89.6)$ & $19(70.4)$ & \\
\hline$\geq 65(32,12.5)$ & $28(12.3)$ & $4(13.3)$ & & $24(10.4)$ & $8(29.6)$ & \\
\hline Alcohol drinking & & & 0.541 & & & 0.086 \\
\hline No $(61,23.7)$ & $55(24.2)$ & $6(20.0)$ & & $51(22.2)$ & $10(37.0)$ & \\
\hline Yes $(196,76.3)$ & $172(75.8)$ & $24(80.0)$ & & 179 (77.8) & $17(63.0)$ & \\
\hline Betel chewing & & & 0.196 & & & 0.194 \\
\hline No $(36,14.0)$ & $34(15.0)$ & $2(6.7)$ & & $30(13.0)$ & $6(22.2)$ & \\
\hline Yes $(221,86.0)$ & 193 (85.0) & $28(93.3)$ & & $200(87.0)$ & $21(77.8)$ & \\
\hline Cigarette smoking & & & 0.048 & & & 0.609 \\
\hline No $(39,15.2)$ & $38(16.7)$ & $1(3.3)$ & & $34(14.8)$ & $5(18.5)$ & \\
\hline Yes $(218,84.8)$ & 189 (83.3) & $29(96.7)$ & & $196(85.2)$ & $22(81.5)$ & \\
\hline Differentiation & & & 0.013 & & & 0.480 \\
\hline Well/moderate $(195,75.9)$ & $178(78.4)$ & $17(56.7)$ & & $176(76.5)$ & $19(70.4)$ & \\
\hline Poor $(62,24.1)$ & $49(21.6)$ & $13(43.3)$ & & $54(23.5)$ & $8(29.6)$ & \\
\hline pT status & & & 0.119 & & & 0.815 \\
\hline pT1-2 (52, 20.2) & $50(22.0)$ & $2(6.7)$ & & $47(20.4)$ & 5 (18.5) & \\
\hline pT3-4 $(205,79.8)$ & $177(78.0)$ & $28(93.3)$ & & $183(79.6)$ & $22(81.5)$ & \\
\hline Depth & & & 0.050 & & & 0.086 \\
\hline$<10 \mathrm{~mm}(61,23.7)$ & $59(26.0)$ & $2(6.7)$ & & $51(22.2)$ & $10(37.0)$ & \\
\hline 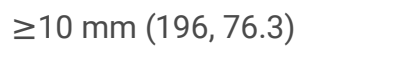 & $168(74.0)$ & $28(93.3)$ & & 179 (77.8) & $17(63.0)$ & \\
\hline Margin & & & 0.046 & & & 0.552 \\
\hline
\end{tabular}

Abbreviations: S, surgery; RT, radiotherapy; CCRT, concurrent chemoradiotherapy. 


\begin{tabular}{|c|c|c|c|c|c|c|}
\hline \multirow[b]{2}{*}{$\leq 4 \mathrm{~mm}(208,80.9)$} & \multicolumn{3}{|c|}{ SUV-nodal-max } & \multicolumn{3}{|c|}{ Lymph node ratio } \\
\hline & $188(82.8)$ & $20(66.7)$ & & $185(80.4)$ & $23(85.2)$ & \\
\hline$>4$ mm $(49,19.1)$ & $39(17.2)$ & 10 (33.3) & & 45 (19.6) & $4(14.8)$ & \\
\hline Perineural invasion & & & 0.132 & & & 0.603 \\
\hline No $(93,36.2)$ & $79(34.8)$ & $14(46.7)$ & & $82(35.7)$ & $11(40.7)$ & \\
\hline Yes $(164,63.8)$ & $148(65.2)$ & $16(53.3)$ & & $148(64.3)$ & $16(59.3)$ & \\
\hline Lymphatic invasion & & & 0.079 & & & 0.100 \\
\hline No $(237,92.2)$ & $196(86.3)$ & $22(73.3)$ & & $198(86.1)$ & $20(74.1)$ & \\
\hline Yes $(20,7.8)$ & $31(13.7)$ & $8(26.7)$ & & $32(13.9)$ & $7(25.9)$ & \\
\hline Vascular invasion & & & 0.674 & & & 0.939 \\
\hline No $(218,84.8)$ & $210(92.5)$ & $27(90.0)$ & & $212(92.2)$ & $25(92.6)$ & \\
\hline Yes $(39,15.2)$ & $17(7.5)$ & $3(10.0)$ & & $18(7.8)$ & $2(7.4)$ & \\
\hline Treatment modality & & & 0.113 & & & 0.004 \\
\hline S plus RT/CCRT $(246,95.7)$ & $219(96.5)$ & $27(90.0)$ & & $223(97.0)$ & $23(85.2)$ & \\
\hline S alone $(11,4.3 \%)$ & $8(3.5)$ & $3(10.0)$ & & $7(3.0)$ & $4(14.8)$ & \\
\hline
\end{tabular}

Most of them were men (94.2\%) and less than 65 years of age (87.5\%). The vast majority (95.7\%) was treated with surgery plus adjuvant therapy. Patients were then stratified according to the optimal cutoff values for SUV-nodal-max and LNR. Compared to patients with SUVnodal-max $<15.9$, those with SUV-nodal-max $\geq 15.9$ had a higher prevalence of the following variables: cigarette smoking, poor differentiation, and margin status $\leq 4 \mathrm{~mm}$. The difference with respect to $\mathrm{DOI} \geq 10 \mathrm{~mm}$ was marginally significant $(p=0.050)$. Compared to patients with LNR $<0.17$, those with SUVnodal-max $\geq 0.17$ had a higher prevalence of the following variables: level IV/V metastases, age of disease onset $\geq 65$ years, and treatment with surgery alone. Supplementary Table 1 summarizes the general characteristics of patients with pN3b disease with or without level IV/V metastases. The distribution of RFs did not show significant differences between the two groups, the only exception being LNR.

\section{Five-year outcomes of patients with pN3b disease}

On the date of the last follow-up, 78 patients with pN3b disease were alive and 179 had died. The median follow-up time for the entire group was 22 months (mean: 50.4 months; SD: 55.0 months; range: 1 - 232 months), whereas the median follow-up for the 78 patients who survived was 97 months (mean: 105.8 months; SD, 55.5 months; range, $24-232$ months). The 5-year outcomes in the entire group of patients with pN3b disease were as follows: LC, 79\%; NC, 72\%; DM, 38\%; DFS, 46\%; DSS, 52\%; and OS, 38\%, respectively.

\section{Multivariable Cox Regression analysis}

We initially assigned the following reference categories $(H R=1)$ : SUV-nodal-max $<15.9$, LNR $<0.17$, absence of level IV/V metastases, female sex, age $<65$ years, negative history for alcohol drinking, negative history for betel chewing, negative history for cigarette smoking, presence of well or moderately differentiated tumors, pT1 - 2 tumor, DOI $<10 \mathrm{~mm}$, margins $>4 \mathrm{~mm}$, absence of perineural invasion, absence of lymphatic invasion, absence of vascular invasion, and treatment with surgery plus adjuvant therapy. Multivariable analyses using a forward stepwise selection procedure identified the following RFs as independently associated with 5-year outcomes: SUV-nodal$\max \geq 15.9$ (NC/DM/DFS/DSS/OS), LNR > 0.17 (NC/DM/DFS/DSS/OS), level IV/V metastases (DM/DFS/DSS/OS), pT3 - 4 tumors (LC/DSS/OS), lymphatic invasion (DFS), positive history for betel chewing (DFS), and treatment with surgery alone (LC/NC/DM/DFS/DSS/OS) (Table 2). The 5-year DFS rates of patients with and without level IV/V metastases were $15 \%$ and $49 \%$, respectively $(p=0.000004$; Fig. 2 -C) 
Table 2

Multivariable analyses of risk factors for 5-year local control, neck control, distant metastasis, and survival rates in patients with 0CSCC and pN3b disease $(n=257)$

\begin{tabular}{|c|c|c|c|c|c|c|c|c|c|c|c|c|}
\hline \multirow[t]{3}{*}{ Risk factor } & \multicolumn{2}{|c|}{ Local control } & \multicolumn{2}{|c|}{ Neck control } & \multicolumn{2}{|c|}{$\begin{array}{l}\text { Distant } \\
\text { metastases }\end{array}$} & \multicolumn{2}{|c|}{$\begin{array}{l}\text { Disease-free } \\
\text { survival }\end{array}$} & \multirow{2}{*}{$\begin{array}{l}\text { Disease- } \\
\text { specific } \\
\text { survival } \\
\text { HR }\end{array}$} & \multicolumn{3}{|c|}{ Overall survival } \\
\hline & HR & $p$ & HR & $p$ & HR & $p$ & HR & $p$ & & $p$ & HR & $p$ \\
\hline & $\begin{array}{l}(95 \% \\
\mathrm{Cl})\end{array}$ & & $\begin{array}{l}(95 \% \\
\mathrm{Cl})\end{array}$ & & $\begin{array}{l}(95 \% \\
\mathrm{Cl})\end{array}$ & & $\begin{array}{l}(95 \% \\
\mathrm{Cl})\end{array}$ & & $\begin{array}{l}(95 \% \\
\mathrm{Cl})\end{array}$ & & $\begin{array}{l}(95 \% \\
\mathrm{Cl})\end{array}$ & \\
\hline \multirow{2}{*}{$\begin{array}{l}\text { SUV-nodal- } \\
\max \geq 15.9 \\
(n=30)\end{array}$} & & \multirow[t]{2}{*}{ ns } & 2.653 & \multirow[t]{2}{*}{0.001} & 2.534 & \multirow[t]{2}{*}{0.001} & 2.491 & \multirow{2}{*}{$\begin{array}{l}<.001 \\
0.00\end{array}$} & 2.090 & \multirow[t]{2}{*}{0.002} & 1.684 & \multirow[t]{2}{*}{0.021} \\
\hline & & & $\begin{array}{l}(1.457- \\
4.832)\end{array}$ & & $\begin{array}{l}(1.499- \\
4.283)\end{array}$ & & $\begin{array}{l}(1.561- \\
3.973)\end{array}$ & & $\begin{array}{l}(1.300- \\
3.362)\end{array}$ & & $\begin{array}{l}(1.081- \\
2.624)\end{array}$ & \\
\hline \multirow{2}{*}{$\begin{array}{l}L N R \geq 0.17 \\
(n=27)\end{array}$} & & \multirow[t]{2}{*}{ ns } & 2.391 & \multirow[t]{2}{*}{0.008} & 2.071 & \multirow[t]{2}{*}{0.012} & 1.884 & \multirow[t]{2}{*}{0.014} & 2.251 & \multirow[t]{2}{*}{0.002} & 1.785 & \multirow[t]{2}{*}{0.013} \\
\hline & & & $\begin{array}{l}(1.262- \\
4.531)\end{array}$ & & $\begin{array}{l}(1.175- \\
3.650)\end{array}$ & & $\begin{array}{l}(1.138- \\
3.117)\end{array}$ & & $\begin{array}{l}(1.362- \\
3.723)\end{array}$ & & $\begin{array}{l}(1.129- \\
2.821)\end{array}$ & \\
\hline \multirow{2}{*}{$\begin{array}{l}\text { Level IV/V } \\
\text { metastases } \\
(\mathrm{n}=27)\end{array}$} & & \multirow[t]{2}{*}{ ns } & & \multirow[t]{2}{*}{ ns } & 2.700 & \multirow{2}{*}{ <. } & 2.356 & \multirow[t]{2}{*}{0.001} & 2.041 & \multirow[t]{2}{*}{0.007} & 1.975 & \multirow[t]{2}{*}{0.003} \\
\hline & & & & & $\begin{array}{l}(1.568- \\
4.648)\end{array}$ & & $\begin{array}{l}(1.444- \\
3.842)\end{array}$ & & $\begin{array}{l}(1.218- \\
3.420)\end{array}$ & & $\begin{array}{l}(1.260- \\
3.095)\end{array}$ & \\
\hline рT3-4 & 2.677 & \multirow[t]{2}{*}{0.041} & & ns & & \multirow[t]{2}{*}{ ns } & & ns & 1.903 & 0.019 & 1.733 & 0.008 \\
\hline$(n=205)$ & $\begin{array}{l}(1.040- \\
6.889)\end{array}$ & & & & & & & & $\begin{array}{l}(1.114- \\
3.251)\end{array}$ & & $\begin{array}{l}(1.154- \\
2.601)\end{array}$ & \\
\hline \multirow{2}{*}{$\begin{array}{l}\text { Lymphatic } \\
\text { invasion } \\
(n=39)\end{array}$} & & \multirow[t]{2}{*}{ ns } & & \multirow[t]{2}{*}{ ns } & & \multirow[t]{2}{*}{ ns } & 1.749 & 0.011 & & ns & & ns \\
\hline & & & & & & & $\begin{array}{l}(1.135- \\
2.694)\end{array}$ & & & & & \\
\hline Betel & & ns & & ns & & ns & 1.868 & 0.044 & & ns & & ns \\
\hline$(\mathrm{n}=221)$ & & & & & & & $\begin{array}{l}(1.017- \\
3.430)\end{array}$ & & & & & \\
\hline Surgery & 7.751 & 0.001 & 3.787 & 0.005 & 2.984 & 0.020 & 4.017 & 0.001 & 4.117 & 0.001 & 5.801 & $<$ \\
\hline$(n=11)$ & $\begin{array}{l}(2.243- \\
26.786)\end{array}$ & & $\begin{array}{l}(1.479- \\
9.695)\end{array}$ & & $\begin{array}{l}(1.186- \\
7.509)\end{array}$ & & $\begin{array}{l}(1.814- \\
8.891)\end{array}$ & & $\begin{array}{l}(1.852- \\
9.154)\end{array}$ & & $\begin{array}{l}(3.036- \\
11.084)\end{array}$ & \\
\hline
\end{tabular}

Abbreviations: SUV, standardized uptake value; LNR, lymph node ratio; HR, hazard ratio; Cl, confidence interval; ns, not significant.

\section{Prognostic scoring system}

On multivariable analysis, we identified SUV-nodal-max $\geq 15.9, \mathrm{LNR} \geq 0.17$, and the presence of level IV/V metastases as independent RFs for 5-year DM, DFS, DSS and OS rates. We therefore devised a four-point prognostic scoring system (range: $0-3$ ) according to the presence or absence of each variable, as follows: 0 for SUV-nodal-max $<15.9$ and 1 for SUV-nodal-max $\geq 15.9 ; 0$ for LNR 0.17 and 1 for $L N R \geq 0.17 ; 0$ for the absence of level IV/V metastases and 1 for the presence of level IV/V metastases. We then constructed the KaplanMeier plots of 5-year outcomes after stratification of patients according to the prognostic score. The number of pN3b patients with a score of $0,1,2$, and 3, was 190, 51, 14, and 2, respectively. Among patients with a score of $1(n=51), 21$ cases had SUV-nodal-max $\geq 15.9,15$ showed a LNR $\geq 0.17$, and 15 had evidence of level IV/V metastases. Three distinct risk groups were then created, as follows: low-risk group (score 0), intermediate-risk group (score 1), and high-risk group (scores 2 - 3).

\section{Five-year survivals in different pN classification}

We found no differences in terms of 5-year DFS rates between patients with pN2a $(64 \%, n=85), p N 2 b(66 \%, n=117)$, and pN2c $(64 \%, n=$ $11)$ disease $(p=0.992)$. Consequently, the three subgroups were combined into a single pN2 group $(n=213)$.

The 5-year rates of pN0/pN1/pN2/pN3b (according to the 2018 AJCC Staging Manual, eight edition) and the subgroups of pN3b deemed at low, intermediate, and high risk according to our scoring system were as follows: DFS, $83 \% / 72 \% / 65 \% / 46 \%$ (Fig. $3-\mathrm{A}$ ) and $54 \% / 26 \% / 0 \%$ (Fig. 3-D); DSS, 92\%/79\%/71\%/52\% (Fig. 3-B) and 59\%/36\%/8\% (Fig. 3-E); OS, 81\%/62\%/54\%/38\% (Fig. 3-C) and 42\%/31\%/6\% (Fig. 3-F); 
all $p<0.001$. As expected, patients in the high-risk group showed the most unfavorable 5 -year outcomes, ultimately confirming that the prognosis of patients with pN3b disease is not homogeneous.

\section{Failure patterns according to the pathological nodal status}

The 5-year rates of pN0/pN1/pN2/pN3b (according to the 2018 AJCC Staging Manual, eight edition) and the subgroups of pN3b deemed at low, intermediate, and high risk according to our scoring system were as follows: LC, $88 \% / 84 \% / 86 \% / 79 \%$ (Fig. 4-A) and $82 \% / 65 \% / 0 \%$ (Fig. 4-D); NC, 93\%/87\%/81\%/72\% (Fig. 4-B) and 78\%/54\%/54\% (Fig. 4-E); DM, 3\%/12\%/20\%/38\% (Fig. 4-C) and 31\%/52\%/89\% (Fig. 4-F); all $p<0.001$ except local control. While the 5 -year rates of patients with $\mathrm{pN} 3 \mathrm{~b}$ disease were clearly distinguishable from those of cases with pNO, pN1, and pN2 disease (Fig. 4A-C), pN3b patients in the high-risk group according to our scoring system had poor outcomes (Fig. 4D-F) - especially in terms of DM (Fig. 4-F).

\section{Discussion}

The 2018 AJCC staging manual has introduced a novel pN3b classification for patients with OCSCC. While the presence of pN3b disease is generally believed to portend poor outcomes, we found that a scoring system based on SUV-nodal-max $\geq 15.9, L N R \geq 0.17$, and the presence of level IV/V metastases improves the prognostic stratification within this subgroup - with high-risk cases (scores $2-3)$ showing the most unfavorable 5-year outcomes. Notably, these patients had a markedly high rate (89\%) of 5-year DM.

Several studies have assessed the prognostic significance of LNR in patients with OCSCC [3-10]. Investigations involving large samples (> 100 cases with pathologically positive nodes) identified optimal cutoff values varying from 0.06 to 0.2 . These variations in the published literature can be explained by differences in the outcomes of interest (e.g., NC, locoregional control, DFS, DSS, and OS) and/or the choice of statistical testing. While the optimal cutoff value for LNR identified in our study (endpoint of interest: 5-year DFS; statistical test: Kaplan-Meier method) was as high as 0.17 , this value is consistent with the published range. Notably, the main advantage of the Kaplan-Meier approach lies in the assessment of clinical events in a time-to-event perspective. This allowed the identification of reliable cutoff values for prognostic stratification in patients with pN3b disease. Importantly, on analyzing the association of LNR with clinical outcomes, we found that this factor was not only associated with survival endpoints, but it also predicted the 5-year DM rates.

Accordingly, for patients with pN3 disease and LNR $\geq 0.17$, we observed a 5-year DM rate of $66 \%$ - with all events occurring within the first two postoperative years.

The prognostic role of the cervical nodal metastases level in patients with OCSCC has been evaluated in a limited number of studies [1, 11, 12]. These investigations found that, compared with patients with level I - III metastases, cases with level IV/V metastases (i.e., lower neck metastasis) have less favorable outcomes in the subgroups of patients with pN1 - 3 disease (DSS) and pN2 disease/ENE (NC, DM, DFS, DSS, and OS). While distant metastases are rarely identified (1\% of all cases) during the preoperative workup of patients with level IV/V metastases, the risk of distant spread at 5 years is as high as $60 \%$ [12]. The 5-year DM rate observed in our study for cases with level IV/V metastases (69\%) is in line with previous observations - albeit being slightly higher as a result of our focus on pN3b disease.

Several investigations involving patients with OCSCC have examined the clinical value of SUV-max calculated from FDG-PET images for preoperative staging [24], identifying second primary tumors [25], allocating patients to adjuvant therapy following tumor removal [26], and assessing response to treatment [27]. Our study is the first to analyze the prognostic role of FDG-PET in patients with OCSCC and pN3 disease. Here, we found that a SUV-nodal-max $\geq 15.9$ was independently associated with less favorable 5-year NC, DM, DFS, DSS, and OS rates. Notably, the 5 -year DM rate of patients with SUV-nodal-max $\geq 15.9$ was as high as $63 \%$, and all events occurred within the first 18 postoperative months. Figure 5 shows an illustrative case of right tongue squamous cell carcinoma (stage: pT4aN3b; LNR: 0.038; no evidence of level IV/V metastasis). The patient's preoperative FDG-PET stage and SUV-nodal-max were TxN2bM0 and 17.4, respectively. A follow-up FDG-PET scan performed 10 months after surgery revealed a TONOM1 stage. The following distant metastatic sites were identified: left hilar and mediastinal lymph nodes, left pleura, left lung, and L3 spine.

The scoring system based on SUV-nodal-max $\geq 15.9, \mathrm{LNR} \geq 0.17$, and the presence of level IV/V metastases allowed an effective prognostic stratification of patients with pN3b disease - with the subgroup of high-risk patients (score 2 - 3) showing dismal outcomes. While being relatively rare $(16 / 257$, i.e., $6 \%$ of all patients with pN3b disease in our study), patients in the high-risk group had a markedly high 5-year DM rate (89\%). Therefore, they should be considered as candidates for novel treatment approaches in clinical trials or receive palliative systemic therapy - rather than traditional CCRT. However, it can be argued that reliable data concerning LRN values cannot be obtained in the preoperative phase. In this scenario, we propose that patients with cN3 disease and concomitant evidence of SUV-nodalmax $\geq 15.9$ and level IV/V metastases on imaging studies (FDG-PET and MRI/CT) should undergo non-surgical treatment to minimize morbidity related to radical tumor excision. 
Several caveats of our study need to be considered. First, its single-center design may have limited the external validity of the results; in addition, the retrospective nature of our investigation could be associated with information bias. Second, the study sample was ethnically homogeneous and all patients were living in a betel quid chewing endemic area; for that reason, more studies are necessary to confirm our findings in Western countries. Third, two different treatment guidelines were used throughout the study (NCCN guidelines before 2008 and CGMH guidelines thereafter). Future analyses stratified by treatment guidelines should work to address this limitation. Finally, on analyzing the variables associated with clinical outcomes, data on clinicopathological RFs were prospectively collected as of 1996; however, FDG-PET imaging was systematically introduced only five years thereafter (2001). Despite these limitations, these data represent a promising step in understanding the prognostic value of SUV-nodal-max, LNR, and the level of cervical nodal metastases in patients with OCSCC and pN3b disease.

On the one hand, our scoring system enables an objective assessment that is suitable for clinical prognostication. On the other hand, the use of our tool has the potential to tailor treatment at the individual level and can find application in clinical trial design.

\section{Declarations}

\section{Funding support}

This study did not receive specific funding.

\section{Conflict of interest statement}

The authors declare no conflicts of interest.

Availability of data and material

listed in the manuscript and supplement files

\section{Authors' contribution}

Study concept and design: Nai-Ming Cheng, Chung-Jan Kang, and Chun-Ta Liao

Data analysis and interpretation: All authors

Manuscript drafting or critical revision for important intellectual content: All authors

Final approval of the manuscript: All authors

Agreement to be accountable for all aspects of the work: All authors

\section{Ethics approval}

The study protocol was granted ethical approval by the local Institutional Review Board (references: CGMH 101-4457B and 202100048B0). The requirement for written consent was waived due to the study design.

\section{Consent to participate and Consent for publication}

This manuscript has not been published or presented elsewhere in part or in entirety and is not under consideration by another journal. All authors have contribution to (1) data analysis and interpretation; (2) manuscript drafting or critical revision for important intellectual content; and (3) final approval of the manuscript. We have read and understood your journal's policies, and we believe that neither the manuscript nor the study violates any of these.

\section{Acknowledgements}

The authors are grateful to the Linkou Chang Gung Memorial Hospital Cancer Center databank and case managers for support and provision of resources.

\section{References}

1. Liao CT, Huang SF, Chen IH, et al. Outcome analysis of patients with pN2 oral cavity cancer. Ann Surg Oncol. 2010;17:1118-26. 
2. Liao CT, Lee LY, Hsueh C, et al. Pathological risk factors stratification in pN3b oral cavity squamous cell carcinoma: Focus on the number of positive nodes and extra-nodal extension. Oral Oncol. 2018;86:188-94.

3. Sayed SI, Sharma S, Rane P, et al. Can metastatic lymph node ratio (LNR) predict survival in oral cavity cancer patients? J Surg Oncol. 2013;108:256-63.

4. Urban D, Gluck I, Pfeffer MR, Symon Z, Lawrence YR. Lymph node ratio predicts the benefit of post-operative radiotherapy in oral cavity cancer. Radiother Oncol. 2013;106:74-9.

5. Künzel J, Mantsopoulos K, Psychogios G, Grundtner P, Koch M, Iro H. Lymph node ratio as a valuable additional predictor of outcome in selected patients with oral cavity cancer. Oral Surg Oral Med Oral Pathol Oral Radiol. 2014;117:677-84.

6. Ebrahimi A, Clark JR, Zhang WJ, et al. Lymph node ratio as an independent prognostic factor in oral squamous cell carcinoma. Head Neck. 2011;33:1245-51.

7. Shrime MG, Bachar G, Lea J, et al. Nodal ratio as an independent predictor of survival in squamous cell carcinoma of the oral cavity. Head Neck. 2009;31:1482-88.

8. Patel SG, Amit M, Yen TC, et al. International Consortium for Outcome Research (ICOR) in Head and Neck Cancer. Lymph node density in oral cavity cancer: results of the International Consortium for Outcomes Research. Br J Cancer. 2013;109:2087-95.

9. Gil Z, Carlson DL, Boyle JO, et al. Lymph node density is a significant predictor of outcome in patients with oral cancer. Cancer. 2009;115:5700-10.

10. Lin NC, Su IH, Hsu JT, Chang YJ, Tsai KY. Comparison of different lymph node staging systems in patients with positive lymph nodes in oral squamous cell carcinoma. Oral Oncol. 2021;114:105146.

11. Liao CT, Lee LY, Huang SF, et al. Outcome analysis of patients with oral cavity cancer and extracapsular spread in neck lymph nodes. Int J Radiat Oncol Biol Phys. 2011;81:930-7.

12. Köhler HF, Kowalski LP. Prognostic impact of the level of neck metastasis in oral cancer patients. Braz J Otorhinolaryngol. 2012;78:15-20.

13. Liao CT, Wang HM, Chang JT, et al. Analysis of risk factors for distant metastases in squamous cell carcinoma of the oral cavity. Cancer. 2007;110:1501-8.

14. Liao CT, Lee LY, Huang SF, et al. Outcome analysis of patients with oral cavity cancer and extracapsular spread in neck lymph nodes. Int J Radiat Oncol Biol Phys. 2011;81:930-7.

15. Edge SB, Byrd DR, Compton CC, et al. AJCC cancer staging manual. 7th ed. New York (NY): Springer-Verlag; 2010.

16. Amin MB, Edge S, Greene F, Byrd DR, Brookland RK, Washington MK, editors. AJCC cancer staging manual. 8th ed.New York: SpringerVerlag; 2017.

17. Seethala RR, Weinreb I, Bullock MJ, et al. Protocol for the Examination of Specimens from Patients with Cancers of the Lip and Oral Cavity. College of American Pathologists; Version: LinOralCavity 4.4.4.1; June 2017.

18. Liao CT, Chang JT, Wang HM, et al. Preoperative [18F] fluorodeoxyglucose positron emission tomography standardized uptake value of neck lymph nodes may aid in selecting patients with oral cavity squamous cell carcinoma for salvage therapy after relapse. Eur $\mathrm{J}$ Nucl Med Mol Imaging. 2009;36:1783-93.

19. Lin CY, Wang HM, Kang CJ, et al. Primary tumor site as a predictor of treatment outcome for definite radiotherapy of advanced-stage oral cavity cancer. Int J Radiat Oncol Biol Phys. 2010;78:1011-9.

20. Fan KH, Wang HM, Kang CJ, et al. Treatment results of postoperative radiotherapy on squamous cell carcinoma of the oral cavity: coexistence of multiple minor risk factors results in higher recurrence rates. Int J Radiat Oncol Biol Phys. 2010;77:1024-9.

21. Wang HM, Liao CT, Chang TC, et al. Biweekly paclitaxel, cisplatin, tegafur, and leucovorin as neoadjuvant chemotherapy for unresectable squamous cell carcinoma of the head and neck. Cancer. 2004;101:1818-23.

22. National Comprehensive Cancer Network Clinical Practice Guidelines in Oncology, Head and Neck Cancers, Version 1.2021. Release date: November 9, 2020. Available at: http://www.nccn.org/. [Accessed April 11, 2021].

23. Lin CY, Fan KH, Lee LY, et al. Precision adjuvant therapy based on detailed pathological risk factors for resected oral cavity squamous cell carcinoma: Long term outcome comparison of CGMH and NCCN guidelines. Int J Radiat Oncol Biol Phys. 2020;106:916-25.

24. $\mathrm{Ng} \mathrm{SH}$, Yen TC, Liao CT, et al. ${ }^{18}$ F-FDG PET and CT/MRI in oral cavity squamous cell carcinoma: A prospective study of 124 patients with histologic correlation. J Nucl Med. 2005;46:1136-43. 
25. Liu FY, Liao CT, Yen TC. Synchronous malignancies in patients with squamous cell carcinoma of the oral cavity. Eur J Nucl Med Mol Imaging. 2011;38:1020-8.

26. Liao CT, Fang KH, Lin CY, et al. Impact of a second FDG-PET scan before adjuvant therapy for the early detection of residual/relapsing tumors in high-risk patients with oral cavity cancer and pathological extracapsular spread. Eur J Nucl Med Mol Imaging. 2012;39:944-55.

27. Liao CT, Wang HM, Huang SF, et al. PET and PET/CT of the neck lymph nodes improves risk prediction in patients with squamous cell carcinoma of the oral cavity. J Nucl Med. 2011;52:180-7.

\section{Figures}

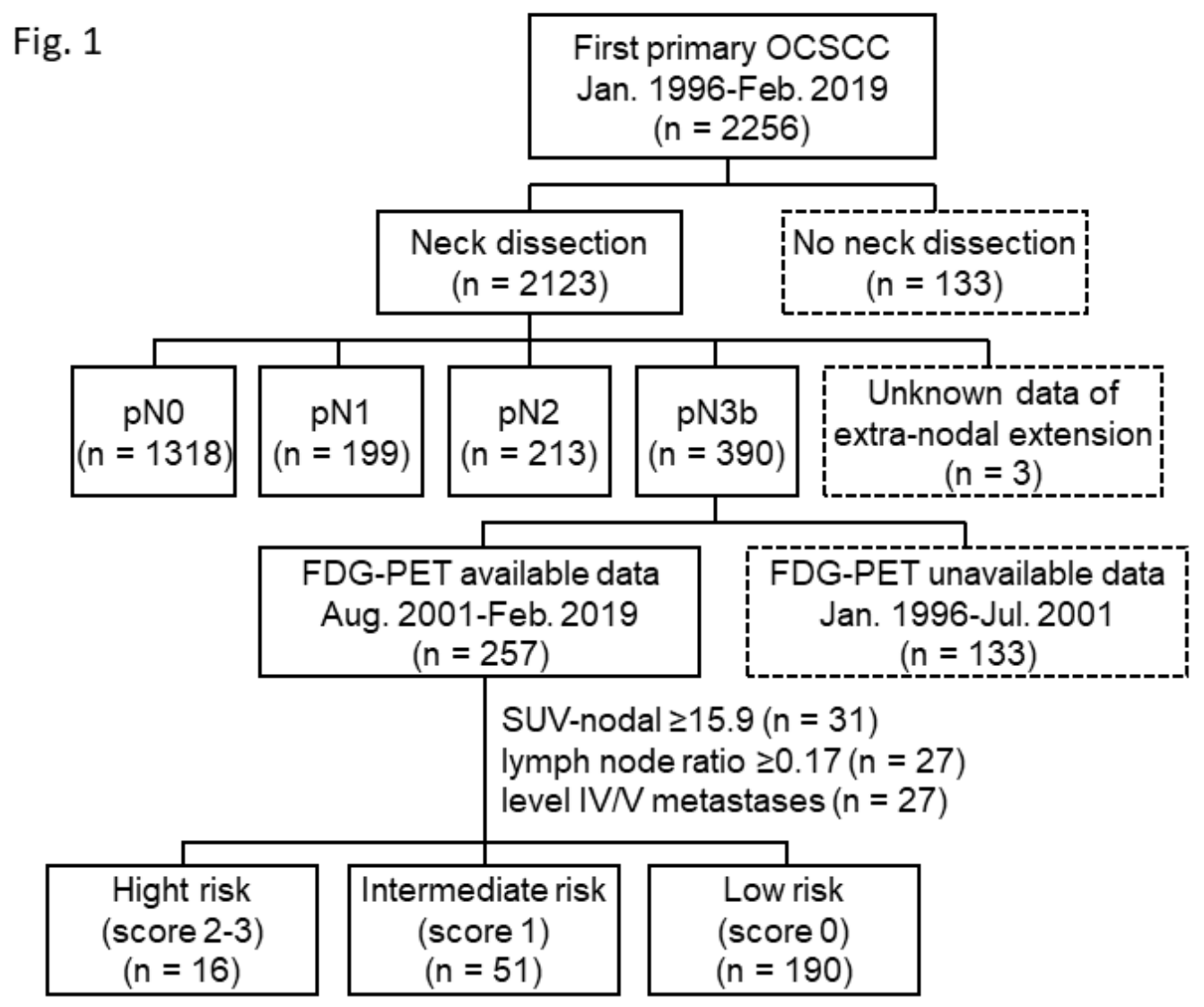

\section{Figure 1}

Flow of patients through the study. 

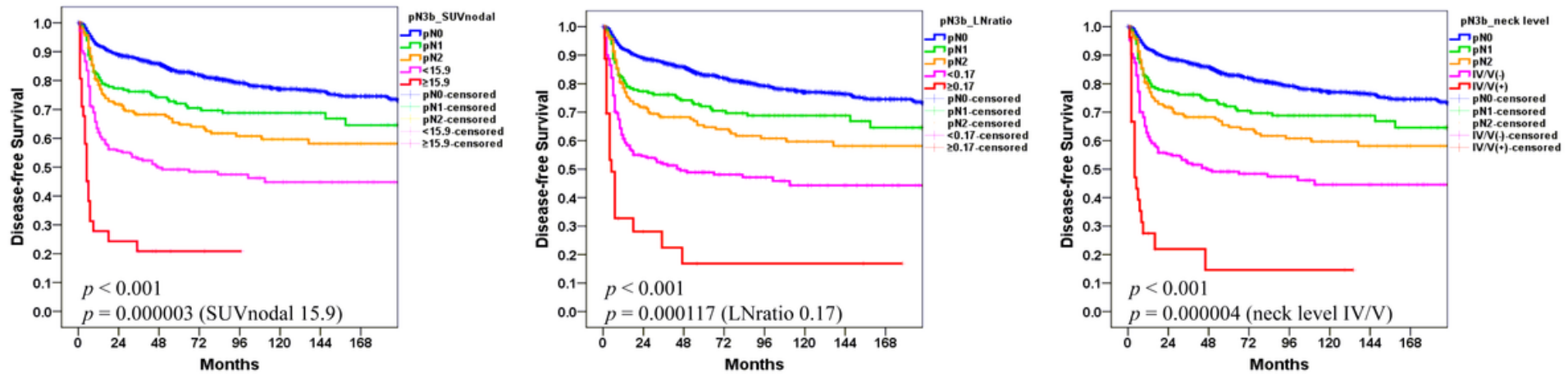

\section{Figure 2}

Kaplan-Meier plots of 5-year disease-free survival in pN0-2 patients and pN3b patients with SUV-nodal-max $<15.9$ versus $\geq 15.9$ (panel A), $\mathrm{LN}$ ratio $<0.17$ versus $\geq 0.17$ (panel $\mathrm{B}$ ), and absence versus presence of level IV/V metastases (panel C).

$3 \mathrm{~A}$

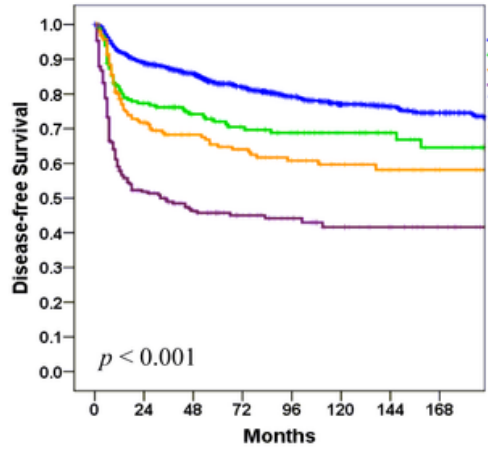

$3 \mathrm{D}$

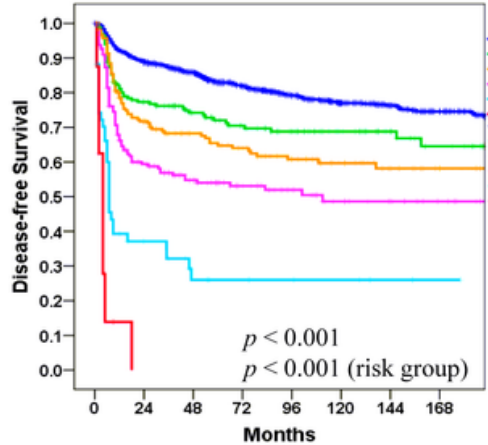

3B

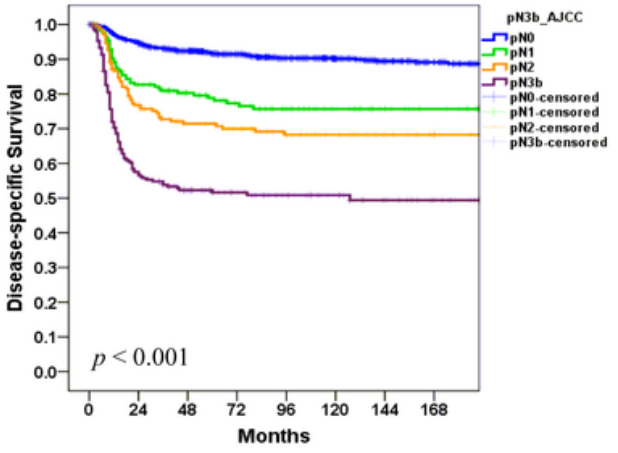

$3 \mathrm{E}$

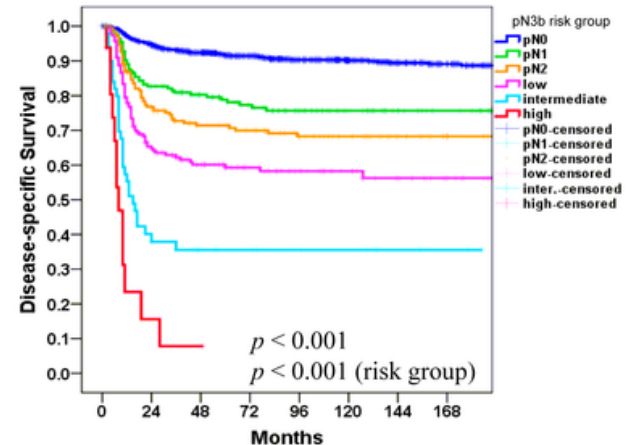

Months
$3 \mathrm{C}$

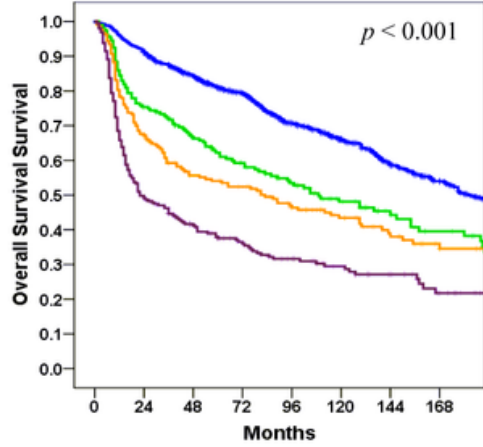

$3 \mathrm{~F}$

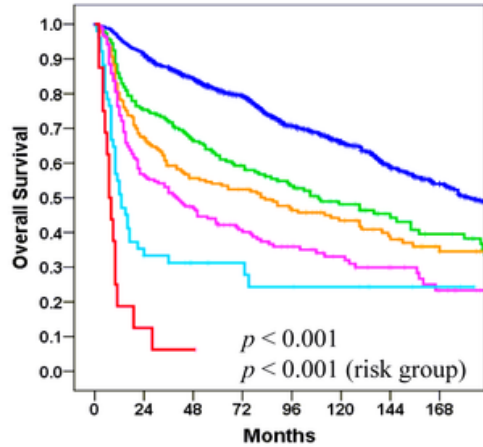

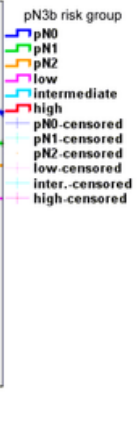

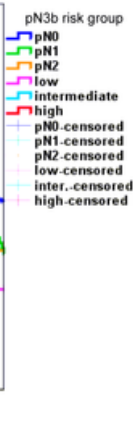

Figure 3

Kaplan-Meier plots of 5-year disease-free survival (panel A), disease-specific survival (panel B), and overall survival (panel C) in patients with pN0, pN1, pN2, and pN3b disease. Kaplan-Meier plots of 5-year disease-free survival (panel D), disease-specific survival (panel E), and overall survival (panel F) in patients with pN0, pN1, pN2 disease, as well as pN3b disease stratified according to the prognostic scoring system (low-, intermediate-, and high-risk) devised in our study. 


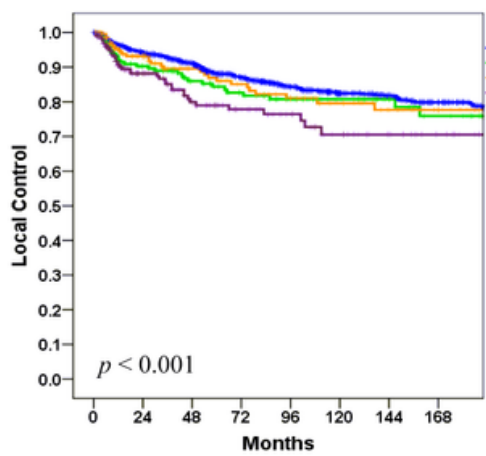

4D

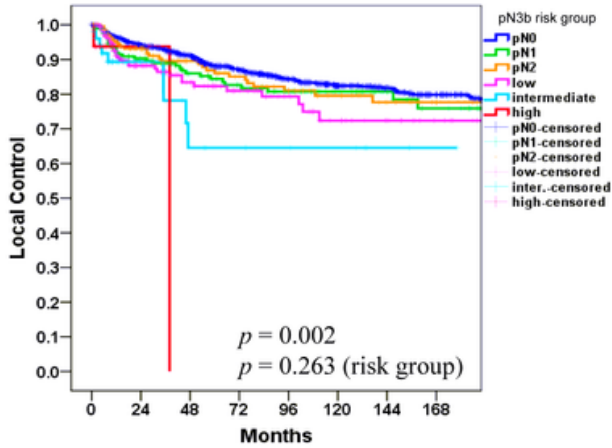

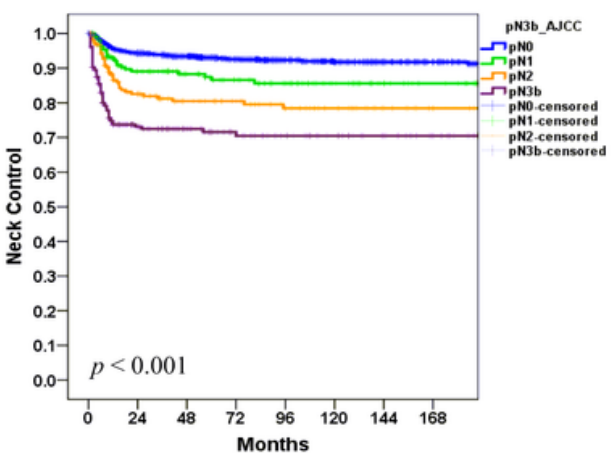

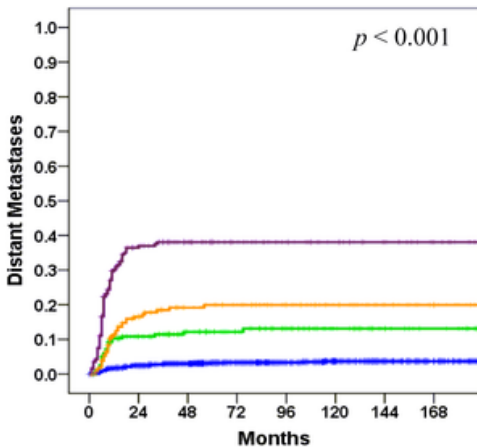

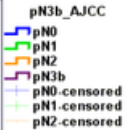

$4 \mathrm{E}$

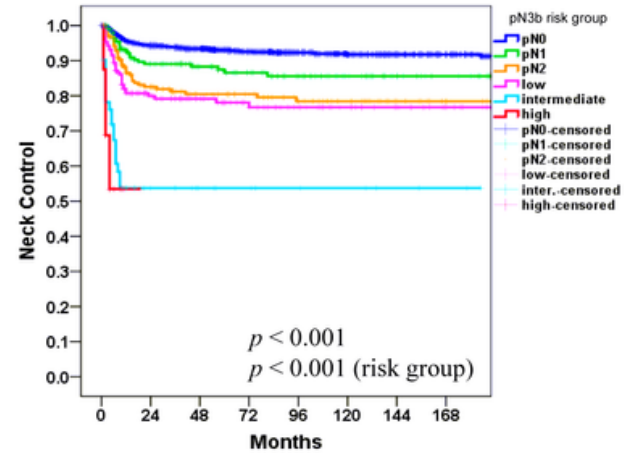

$4 \mathrm{~F}$

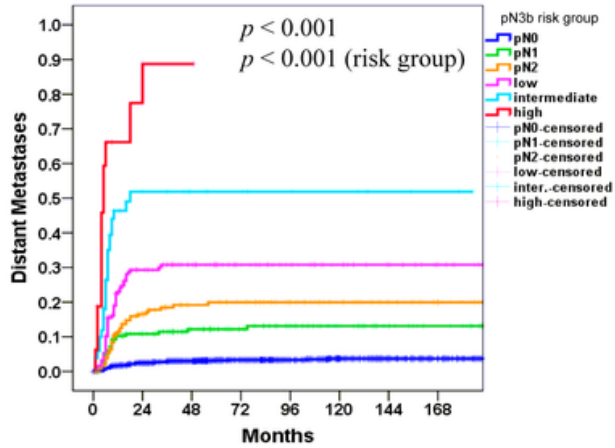

\section{Figure 4}

Kaplan-Meier plots of 5-year local control (panel A), neck control (panel B), and distant metastases (panel C) in patients with pN0, pN1, pN2, and pN3b disease. Kaplan-Meier plots of 5-year local control (panel D), neck control (panel E), and distant metastases (panel F) in patients with pN0, pN1, pN2 disease, as well as pN3b disease stratified according to the prognostic scoring system (low-, intermediate-, and high-risk) devised in our study.

Fig. 5
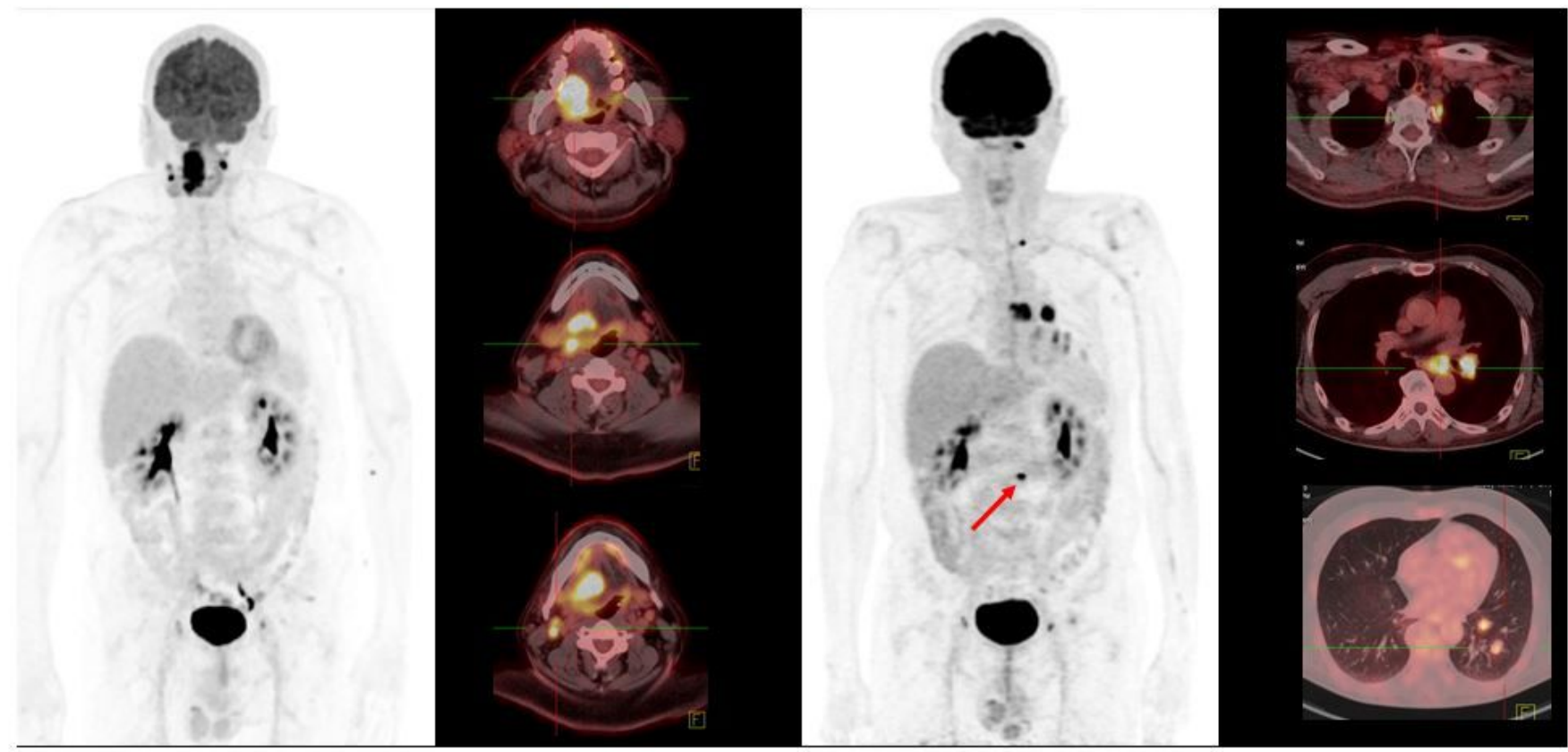


\section{Figure 5}

Illustrative case of right tongue squamous cell carcinoma (stage: pT4aN3b). The patient showed right level I (1/20), right level II (3/30), and left level II (1/5) cervical lymph node metastases. Of the five metastatic nodes, four were characterized by extracapsular extension (ENE). All of the nodes with ENE were located in the right neck. According to preoperative FDG-PET imaging (right image), disease stage was TxN2bM0. The tumor and nodal maximum SUV values were 23.3 (score 4) and 17.4 (right level II, score 4), respectively. A follow-up FDG-PET scan performed 10 months after surgery (left image) revealed a TONOM1 stage. The following distant metastatic sites were identified: left hilar and mediastinal lymph nodes (score 4), left pleura (score 4), left lung (score 4), and L3 spine (score 4, arrowhead).

\section{Supplementary Files}

This is a list of supplementary files associated with this preprint. Click to download.

- R1SUPPLTABLE.doc 